\title{
Clinical Study \\ Influence of Sleeve Gastrectomy on NASH and Type 2 Diabetes Mellitus
}

\section{W. K. Karcz, ${ }^{1}$ D. Krawczykowski, ${ }^{2}$ S. Kuesters, ${ }^{1}$ G. Marjanovic, ${ }^{1}$ B. Kulemann, ${ }^{1}$ H. Grobe, ${ }^{1}$ I. Karcz-Socha, ${ }^{3}$ U. T. Hopt, ${ }^{1}$ W. Bukhari, ${ }^{4}$ and J. M. Grueneberger ${ }^{1}$}

\author{
${ }^{1}$ Department of General and Visceral Surgery, Albert-Ludwigs-University, Hugstetter Straße 55, 79106 Freiburg, Germany \\ ${ }^{2}$ Department of Surgery, Champain de Chalons, 2 Rue Charles Simon, 51308 Vitry-le-France, Frankreich, France \\ ${ }^{3}$ Department of Physiology, Silesian Medical University, Jordana 19, 41-800 Zabrze, Poland \\ ${ }^{4}$ Department of Laparoscopic Surgery, International Medical Center, P.O. Box 2173, Jeddah 21451, Saudi Arabia
}

Correspondence should be addressed to J. M. Grueneberger, jodok.grueneberger@uniklinik-freiburg.de

Received 31 August 2010; Accepted 26 October 2010

Academic Editor: Francesco Saverio Papadia

Copyright ( $) 2011$ W. K. Karcz et al. This is an open access article distributed under the Creative Commons Attribution License, which permits unrestricted use, distribution, and reproduction in any medium, provided the original work is properly cited.

\begin{abstract}
Background. Nonalcoholic fatty liver disease is present in up to $85 \%$ of adipose patients and may proceed to nonalcoholic steatohepatitis (NASH). With insulin resistance and obesity being the main risk factors for NASH, the effect of isolated sleeve gastrectomy (ISG) on these parameters was examined. Methods. 236 patients underwent ISG with intraoperative liver biopsy from December 2002 to September 2009. Besides demographic data, pre-operative weight/BMI, HbA1c, AST, ALT, triglycerides, HDL and LDL levels were determined. Results. A significant correlation of NASH with higher HbA1c, AST and ALT and lower levels for HDL was observed $(P<.05,<.0001,<.0001,<.01$, resp. $)$. Overall BMI decreased from $45.0 \pm 6.8$ to $29.7 \pm 6.5$ and $31.6 \pm$ $4.4 \mathrm{~kg} / \mathrm{m}^{2}$ at 1 and 3 years. An impaired weight loss was demonstrated for patients with NASH and patients with elevated HbA1c (plateau $28.08 \mathrm{~kg} / \mathrm{m}^{2}$ versus $29.79 \mathrm{~kg} / \mathrm{m}^{2}$ and $32.30 \mathrm{~kg} / \mathrm{m}^{2}$ versus $28.79 \mathrm{~kg} / \mathrm{m}^{2}$, resp.). Regarding NASH, a significant improvement of AST, ALT, triglyceride and HDL levels was shown $(P<.0001$ for all $)$. A resolution of elevated HbAlc was observed in 21 of 23 patients. Summary. NASH patients showed a significant loss of body weight and amelioration of NASH status. ISG can be successfully performed in these patients and should be recommended for this subgroup.
\end{abstract}

\section{Introduction}

Nonalcoholic fatty liver disease (NAFLD) describes a wide spectrum of liver pathologies from simple steatosis to steatohepatitis (NASH) and cirrhosis [1]. NAFLD has a prevalence of $10 \%$ to $24 \%$ in the general population. Its prevalence in obese persons is between $50 \%$ and $85 \%$, and 65-90\% in those with Type 2 Diabetes mellitus (T2DM) [2].

For patients diagnosed with NASH, 15-20\% may develop cirrhosis [3]. Given the fact that patients with NASH can enter a final cirrhotic pathway, it is not surprising that NASH appears to portend an increased risk of hepatocellular carcinoma [4].

Liver function test (lft) abnormalities are common in patients with NAFLD, with elevations in aspartate aminotransferase (AST) and alanine aminotransferase (ALT) usually no greater than four times the upper limit of normal and a variable AST/ALT ratio, although ALT usually predominates [5]. Several noninvasive models have been established to differentiate steatosis from NASH. It is not clear to date whether biopsy is needed for the diagnosis of NASH [6].

The pathophysiology of NASH is regarded as a continuous process with two major factors. The first factor is considered to be insulin resistance resulting in steatosis by increased elaboration of free fatty acids (FFA) that are absorbed by the liver. The second factor is a series of complex interactions between hepatocytes, stellate cells, adipose cells, Kupffer cells, inflammatory mediators, and reactive oxygen species that result in inflammation (NASH) or cirrhosis [7]. Here, the adipose tissue, whose function goes far beyond gathering energy supplies, seems to play a key role, being an essential endocrine organ producing a number of proteins demonstrating auto- and paracrine effects [8]. 
Unlike other chronic diseases such as T2DM, there are no formal treatment algorithms for patients with NAFLD. Currently, management of NAFLD consists of modifying the underlying risk factors including medical therapy [9-14]. To induce weight loss in NASH patients, bariatric surgery is discussed as a potential alternative.

Sleeve gastrectomy was first introduced by Hess and Marceau as a restrictive component of biliopancreatic diversion with duodenal switch; it was initially not intended as a standard single procedure [15]. Reported weight loss after isolated sleeve gastrectomy (ISG) is good and ranges from $33 \%$ to $83.3 \%$ of EWL at the 1 -year followup [16, 17]. Today sleeve gastrectomy is performed as a stand-alone procedure with a low operative risk. The most common complication is staple line insufficiency and staple line bleeding [18]. Although it is mainly a restrictive procedure, resolution of T2DM occurs in $50 \%$ to over $94 \%$ of patients after ISG by 1-year followup $[19,20]$.

The impact of bariatric surgery on NASH has been examined for several bariatric interventions such as gastric bypass or duodenal switch operations. Based on the data reported for isolated sleeve gastrectomy regarding weight loss and amelioration of T2DM, we examined the effect of ISG on $\mathrm{NASH}$ and NASH-related comorbidities using clinical and biological data.

\section{Material and Methods}

2.1. Patients. A review of prospectively collected data was conducted for 236 consecutive patients, undergoing ISG at Polyclinique Priollet, Châlons en Champagne, France from December 2002 to September 2009. Laparoscopic Sleeve Gastrectomy was performed as previously described using a 36 French calibration tube. Methylene blue was injected intraoperatively to check for leakage [21].

Data collected included demographic data, preoperative weight/BMI, HbAlc levels, liver functions tests (AST and ALT), and lipid profiling (triglycerides, HDL, and LDL). Early followup examinations were conducted at 6 weeks and 6 months after ISG, the later followup time span was yearly. All data were entered prospectively into a custom-designed database. Approval for the surgery and prospective audit was granted by the institution's clinical board.

2.2. Histological Assessment. Liver biopsy was conducted percutaneously during ISG under laparoscopic guidance using a 14-gauge $200 \mathrm{~mm}$ Tru Cut Biopsy needle (CareFusion). The biopsies were taken from the central part of the left lobe of liver. All liver biopsy specimens were stained with H\&E, PAS, Masson trichrome, Perls and Van Gieson. For the assessment of liver histology, one pathologist and a second observer evaluated all biopsies according to the semiquantitative scoring system for NAFLD proposed by Brunt et al. [22].

2.3. Statistics. Statistical analysis was conducted using Prism 5 for Mac OS X (GraphPad Software, Inc.)

One and two-way ANOVA were used to compare and evaluate biological data. Spearman's correlation was applied
TABle 1: Patient characteristics.

\begin{tabular}{lc}
\hline Variable & \\
\hline Number of patients & 236 \\
female $\%$ & 87 \\
Age at operation (years) & $37.9 \pm 10.0^{*}$ \\
Weight $(\mathrm{kg})$ & $119.5 \pm 19.4^{*}$ \\
BMI kg/m ${ }^{2}$ & $45.0 \pm 6.8^{*}$ \\
Liver biopsy & \\
$\quad$ NASH & 87 \\
$\quad$ normal liver histology & 35 \\
$\quad$ mean degree of steatosis & $33.6 \pm 24.7^{*}$ \\
HbA1c $>6.5 \%$ & 23 \\
\hline
\end{tabular}

${ }^{*}$ mean \pm standard deviation.

to asses a correlation between two variables. A $P$-value $<.05$ was considered significant.

\section{Results}

Isolated sleeve gastrectomy was performed in 236 patients with a mean BMI of $45 \pm 6.8 \mathrm{~kg} / \mathrm{m}^{2}$ (Table 1 ). In order to assess liver histology, intraoperative liver biopsy was accomplished in all patients. From the 223 histologic samples that could be finally evaluated, diagnosis was as follows: 35 patients with normal liver, 77 with steatosis, 87 with NASH, 18 with fibrosis, 3 with cirrhosis, 2 with siderosis, and one patient with chronic hepatitis (Table 1). Patient followup was conducted up to 3 years with a median overall followup of 12 months. Among the clinical and biological data analysed, NASH was significantly correlated with higher HbA1c, AST, and ALT levels and lower levels for HDL (Spearman's correlation <.05, <.0001, <.0001, <.01, resp.). Elevated ALT levels could only be detected in 2 of 33 patients with normal liver histology, but in 33 of 65 patients with NASH (Fisher's exact test $P<.0001$ ). Other biological data were similar in both groups (Table 3 ). Interestingly, there was a significant correlation between a progressing liver disease from normal liver histology over steatosis to NASH and rising HbA1c levels (Pearson's correlation $P<.01$ ).

Our main objective was to determine if NASH has an impact on the primary outcome after ISG, and equally important to analyze the effect of ISG on NASH.

Overall, ISG led to substantial weight loss with a BMI reduction of $44 \%$ within 1 year (Table 2). Comparing patients with normal liver histology to patients diagnosed with NASH, a significant reduction of body weight was seen in both groups with a marginally larger preoperative BMI in NASH patients $\left(44.2 \pm 5.6 \mathrm{~kg} / \mathrm{m}^{2}\right.$ versus $45.9 \pm 8.3 \mathrm{~kg} / \mathrm{m}^{2}$; NS). Nonlinear regression analysis suggested equal initial weight loss in both groups with an impaired reduction of BMI for NASH patients beginning after the 9-month followup (plateau $28.08 \mathrm{~kg} / \mathrm{m}^{2}$ versus $29.79 \mathrm{~kg} / \mathrm{m}^{2}$, Figure 1). Overall, patients with normal liver histology presented with a significantly better weight reduction (Two-way ANOVA $P<$ $.05)$. Determining HbA1c levels, 23 patients were considered diabetic prior to the operation. Histologic evaluation of 


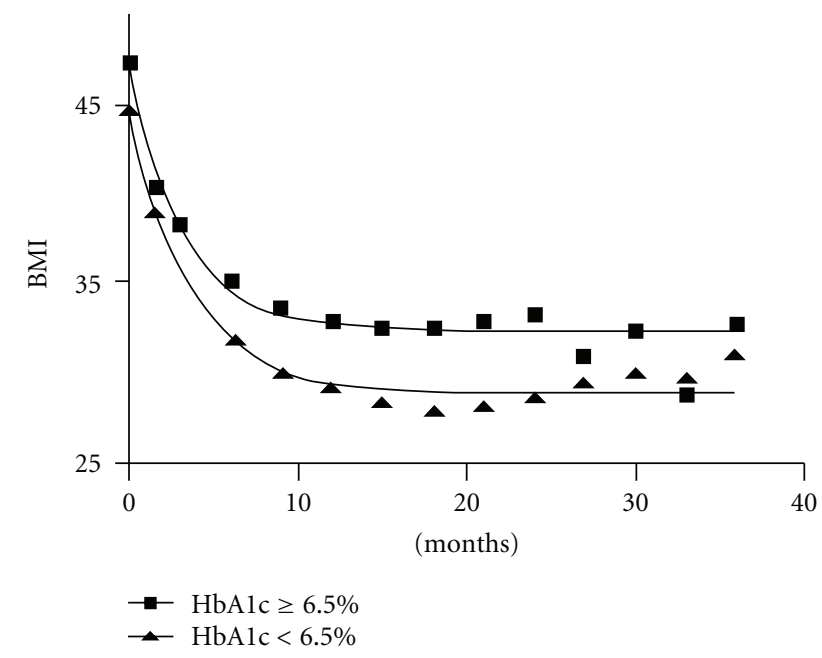

Figure 1: Course of BMI after ISG for patients with HbAlc $\geq$ $6.5 \%$ versus patients with $\mathrm{HbAlc}<6.5 \%$. Nonlinear regression $95 \%$ CI plateau 31.12 to $33.49 \mathrm{~kg} / \mathrm{m}^{2}$ versus 28.31 to $29.26 \mathrm{~kg} / \mathrm{m}^{2}$, respectively, two-way ANOVA $P<.0001$.

this subgroup revealed only 2 patients with normal liver histology, 4 with steatosis, 14 with NASH, 1 with cirrhosis, 1 with fibrosis, and 1 with chronic hepatitis. There was a highly significant difference in weight reduction compared to nondiabetic patients, (two-way ANOVA $P<.001$ ). Nonlinear regression analysis revealed a broader difference in plateau level comparing NASH patients to patients with normal liver histology (plateau for $\mathrm{HbAlc}>6.5 \% 32.30 \mathrm{~kg} / \mathrm{m}^{2}$ versus $\mathrm{HbAlc} \leq 6.5 \% 28.79 \mathrm{~kg} / \mathrm{m}^{2}$, Figure 2).

Regarding biological data, ISG could significantly improve HbAlc levels in all patients (Kruskal-Wallis $P<$ .0001 , Table 2). In the 23 patients diagnosed with diabetes prior to sleeve gastrectomy, median $\mathrm{HbAl}$ c levels normalized within 12 months (HbAlc 7.1\% versus 5.6\%, resp.). A significant reduction of $\mathrm{HbAlc}$ levels could also be shown for NASH patients (Kruskal-Wallis $P<.01$ ). In patients who presented with normal liver histology, sleeve gastrectomy had no effect on $\mathrm{HbAl}$; , however, mean $\mathrm{HbAlc}$ was not above the cutoff level of $6.5 \%$ at any given time point (Table 3 ).

Regarding lipids, ISG had a significant impact on triglyceride levels. Overall mean triglyceride levels could be reduced by $37.5 \%$ after 1 year (Table 2). In patients with NASH, initial triglyceride levels were higher than in patients with normal liver histology $(1.8 \pm 1.4 \mathrm{mmol} / \mathrm{L}$ versus $1.4 \pm 0.7 \mathrm{mmol} / \mathrm{L})$. Although a significant reduction of triglycerides was seen in both groups, NASH had a significantly impairing impact on triglyceride levels (two-way ANOVA $P<.05$, Table 3 ).

Overall, analysis of the remaining lipid profile showed no influence of ISG on cholesterol, HDL, or LDL levels (Table 2). Subgroup analysis revealed no significant impact on either cholesterol or LDL levels. HDL levels again remained unchanged for patients with normal liver histology. In contrast, NASH patients presented a significant improvement of HDL (Kruskal-Wallis $P<.0001$, Table 3 ).

Transaminase levels were examined as a representative test for liver function and liver injury. In preoperative data,

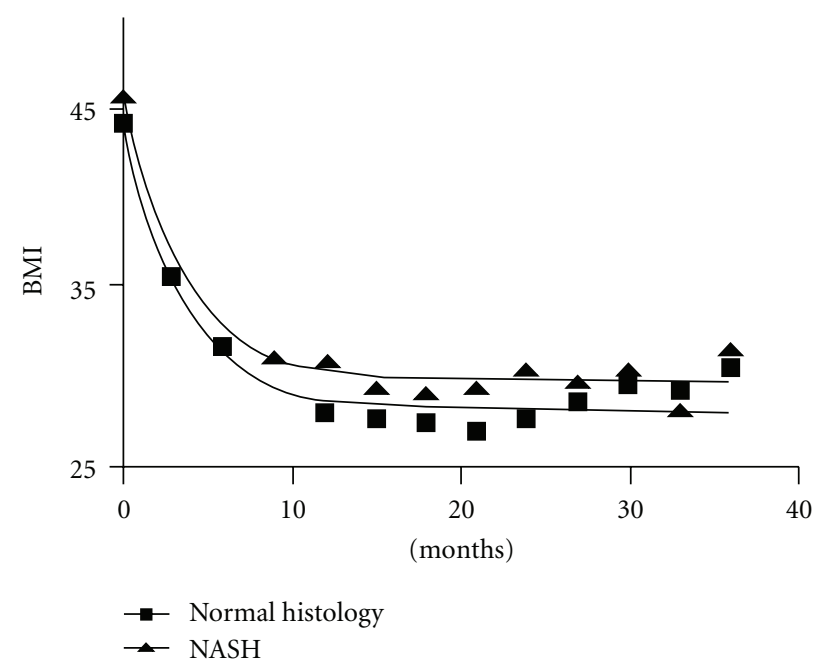

FIGURE 2: Course of BMI after ISG for patients with normal liver histology versus patients with NASH. Nonlinear regression $95 \%$ CI plateau 27.27 to $28.89 \mathrm{~kg} / \mathrm{m}^{2}$ versus 28.69 to $30.89 \mathrm{~kg} / \mathrm{m}^{2}$, respectively, two-way ANOVA $P<.05$.

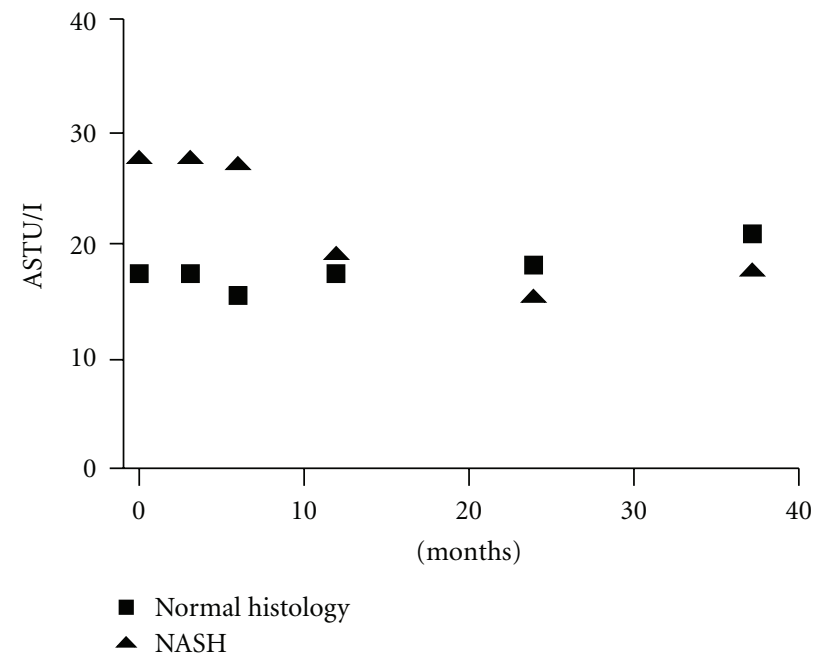

Figure 3: Course of AST levels over a 3-year followup. A significant reduction of AST levels could be shown for NASH patients (ANOVA $P<.01)$.

a strong correlation could be seen between high transaminase levels and histologically diagnosed NASH (Spearman's correlation $P<.0001$ and $<.0001$ for ALT and AST, resp.). For patients with NASH, transaminase levels could be reduced by over $50 \%$ within 6 months, achieving similarity to the levels of patients with normal histology. This effect remained stable throughout the followup period. For patients with normal histology, transaminase levels were low and remained unchanged after sleeve gastrectomy (Figure 3).

\section{Discussion}

In the present study, we report a mean BMI loss of $15.3 \mathrm{~kg} / \mathrm{m}^{2}$ and $13.4 \mathrm{~kg} / \mathrm{m}^{2}$ at 12 and 39 months after ISG. This goes 
TABLE 2: Overall clinical and biological data at 1- and 3-year followup.

\begin{tabular}{|c|c|c|c|c|}
\hline Variable & Before surgery & 1 year after surgery & 3 years after surgery & $P$ value \\
\hline $\mathrm{BMI} \mathrm{kg} / \mathrm{m}^{2}$ & $45.0 \pm 6.8$ & $29.7 \pm 6.5$ & $31.6 \pm 4.4$ & $<.0001$ \\
\hline HbA1c \% & $5.8 \pm 1.1$ & $5.3 \pm 0.5$ & $5.4 \pm 1.2$ & $<.0001$ \\
\hline AST level U/L & $22.7 \pm 11.9$ & $18.14 \pm 9.9$ & $20.0 \pm 10.8$ & $<.0001$ \\
\hline ALT level U/L & $34.2 \pm 21.2$ & $19.8 \pm 14.8$ & $23.01 \pm 17.2$ & $<.0001$ \\
\hline Triglyceride level mmol/L & $1.6 \pm 1.1$ & $1.0 \pm 0.4$ & $1.0 \pm 0.5$ & $<.0001$ \\
\hline Cholesterol level mmol/L & $5.1 \pm 1.1$ & $5.1 \pm 1.0$ & $5.1 \pm 1.3$ & NS \\
\hline $\mathrm{LDL}$ level mmol/L & $3.1 \pm 0.8$ & $3.1 \pm 1.0$ & $3.1 \pm 1.6$ & NS \\
\hline HDL level mmol/L & $1.3 \pm 0.5$ & $1.26 \pm 0.5$ & $1.6 \pm 0.4$ & $<.0001$ \\
\hline
\end{tabular}

Figures presented as mean \pm standard deviation; $P$ value determined with Kruskal-Wallis test.

TABLE 3: Separate consideration of clinical and biological data at 1- and 3-year followup for patients with NASH and normal liver histology.

\begin{tabular}{|c|c|c|c|c|c|c|c|c|c|}
\hline \multirow{2}{*}{ Variable } & \multicolumn{2}{|c|}{ Before surgery } & \multicolumn{2}{|c|}{1 year after surgery } & \multicolumn{2}{|c|}{3 years after surgery } & \multicolumn{2}{|c|}{$P$ value } & \multirow{2}{*}{$P$ difference } \\
\hline & Normal liver & NASH & Normal liver & NASH & Normal liver & NASH & Normal liver & NASH & \\
\hline $\mathrm{BMI} \mathrm{kg} / \mathrm{m}^{2}$ & $44.2 \pm 5.6$ & $45.9 \pm 8.3$ & $28.2 \pm 4.9$ & $30.8 \pm 8.8$ & $30.3 \pm 5.6$ & $30.8 \pm 5.3$ & $<.0001$ & $<.0001$ & $<.05$ \\
\hline $\mathrm{HbA} 1 \mathrm{c} \%$ & $5.4 \pm 0.5$ & $6.1 \pm 1.4$ & $5.2 \pm 0.4$ & $5.4 \pm 0.4$ & $4.9 \pm 0.4$ & $5.2 \pm 0.5$ & NS & $<.01$ & $<.001$ \\
\hline AST level U/L & $17.9 \pm 4.8$ & $27.9 \pm 14.4$ & $18 \pm 11.2$ & $19.2 \pm 9.3$ & $19.2 \pm 11.0$ & $17.2 \pm 2.4$ & NS & $<.01$ & NS \\
\hline ALT level U/L & $23.9 \pm 9.1$ & $42.1 \pm 25.3$ & $25.8 \pm 27.3$ & $17.5 \pm 7.3$ & $22.0 \pm 16.1$ & $12.2 \pm 1.9$ & NS & $<.0001$ & NS \\
\hline Triglyceride level mmol/l & $1.4 \pm 0.7$ & $1.8 \pm 1.4$ & $0.9 \pm 0.3$ & $1.0 \pm 0.3$ & $0.8 \pm 0.3$ & $0.9 \pm 0.4$ & $<.05$ & $<.0001$ & $<.05$ \\
\hline Cholesterol level mmol/L & $5.3 \pm 0.8$ & $5.0 \pm 1.0$ & $5.7 \pm 1.5$ & $5.0 \pm 0.8$ & $4.8 \pm 1.1$ & $5.2 \pm 0.9$ & NS & NS & NS \\
\hline $\mathrm{LDL}$ level mmol/L & $3.3 \pm 0.7$ & $3.0 \pm 0.8$ & $3.6 \pm 0.9$ & $3.1 \pm 0.8$ & $2.7 \pm 1.1$ & $3.3 \pm 0.7$ & NS & NS & NS \\
\hline HDL level mmol/L & $1.4 \pm 0.4$ & $1.2 \pm 0.4$ & $1.7 \pm 0.7$ & $1.5 \pm 0.3$ & $1.5 \pm 0.5$ & $1.5 \pm 0.3$ & NS & $<.0001$ & NS \\
\hline
\end{tabular}

Figures presented as mean \pm standard deviation; separate $P$ values for NASH and normal liver histology were determined with Kruskal-Wallis test, $P$ difference determined with two-way ANOVA.

along with data recently published on 200 patients with isolated sleeve gastrectomy, reporting a weight loss from $45.5 \mathrm{~kg} / \mathrm{m}^{2}$ to $30.6 \mathrm{~kg} / \mathrm{m}^{2}$ and $31.7 \mathrm{~kg} / \mathrm{m}^{2}$ at 12 and 18 months, respectively, using a $48 \mathrm{Fr}$ calibration tube [23]. A Greek centre, at which a 38 Fr bougie was used, reported a comparable weight loss from $45.3 \mathrm{~kg} / \mathrm{m}^{2}$ to $30.5 \mathrm{~kg} / \mathrm{m}^{2}$ for 246 patients undergoing ISG [24]. In a series of 163 patients undergoing ISG using a 36 French calibration tube, weight loss from $45.9 \mathrm{~kg} / \mathrm{m}^{2}$ to $33.1 \mathrm{~kg} / \mathrm{m}^{2}$ and $31.6 \mathrm{~kg} / \mathrm{m}^{2}$ was presented at 1- and 2-year followup. Bougie size does not seem to have an impact on initial weight loss; however, sleeve dilatation and a consequent regain of weight occur for larger bougies $[18,25]$.

A diabetic metabolism with high insulin levels and thus inadequate glucose and lipid homoeostasis is expected to lead to poor weight loss after obesity surgery. This is supported by evidence found by Caiazzo et al., who reported an impaired weight loss for diabetic patients after laparoscopic gastric banding (LAGB) at one-year followup [26]. Schauer et al. showed a comparable effect on EWL\% after laparoscopic gastric bypass (LGB) [27]. Similar findings are presented by Mathurin et al., who reported a significantly lower weight loss for patients presenting with insulin resistance prior to obesity surgery (laparoscopic gastric banding and bypass) [28]. However, these findings remain controversial, as others report no difference regarding weight loss in their subgroup analysis [29].

Our data suggest a significantly impaired weight loss for patients with elevated HbAlc levels at baseline despite a good resolution of $\mathrm{HbAlc}$, suggesting elevated $\mathrm{Hb} 1 \mathrm{Ac}$ level as a predictor for poor weight loss. However, weight loss is still highly significant in this group.

Comparing weight loss of patients diagnosed with NASH during the operation to patients with normal liver histology, an impairment in loss of BMI is evident. This phenomenon can partly be explained by the impaired weight loss of diabetic patients; however, only 14 of 87 patients with NASH showed an HbA1c above $6.5 \%$. Certainly, changes in adipokine profiling play a pivotal role in NASH pathology. New studies simultaneously evaluating NASH and adipokine profiling should further evaluate this context.

Dixon et al. examined the effect of LAGB on the histopathological features of NAFLD, performing two consecutive liver biopsies and determining plasma aminotransferase concentrations at a median followup time of 30 months. This group could show a significant correlation of aminotransferase concentrations and presence of NASH at the first and second biopsies. An aminotransferase decrease of $38 \%$ could best and significantly predict the change in NASH status [30].

Ulitzky et al. identified diabetes, ALT, and triglyceride levels as independent risk factors for NASH in multivariate analysis [31]. In our cohort, we could show a strong correlation of preoperatively determined ALT and HbAlc levels with histologically confirmed NASH. A limitation of our study is the lack of a second liver biopsy, which prevents clearly defining the effect of sleeve gastrectomy on liver histology. In the NASH subgroup, however, we could 
show a significant reduction of triglyceride, HbA1c, and ALT levels, resulting in a significant reduction of NASH incidence, when applying the Ulitzky model [32]. Furthermore, patients diagnosed with NASH presented with a $39 \%$ decrease in AST level.

Lacking a distinct algorithm, NASH therapy is mainly based on the reduction of underlying risk factors. With obesity being the single most significant risk factor, weight loss surgery is widely discussed for the management of NASH. A recently conducted Cochrane research concerning this subject analyzed 21 independent studies. Among the bariatric procedures used, the majority of the studies were conducted with Roux-en-Y gastric bypass (13) and adjustable gastric banding (6). Regarding histological outcomes, eighteen studies reported a significant improvement in the degree of steatosis and eleven studies reported an improvement in histological markers of inflammation. In 11 of 16 studies lfts improved in the course of followup, aggravation could not be observed [33]. In concordance with the data presented above, our data suggest amelioration of NASH after ISG.

An amelioration of T2DM after ISG is currently under intensive discussion. Abbatini et al. showed a $80.9 \%$ resolution of T2DM after ISG over a 3-year followup [34]. In a series of 39 consecutive patients with preoperatively diagnosed T2DM undergoing ISG, Vidal et al. presented a resolution of HbA1c levels below $6.5 \%$ in $94.6 \%$ at 1year followup. Furthermore, they could show a 50\% to $80 \%$ resolution of metabolic syndrome associated figures such as triglyceride and HDL levels, suggesting ISG as a successful strategy for the management of different cardiovascular risk components of the metabolic syndrome [20]. In our subgroup analysis of patients with elevated $\mathrm{HbA1c}$, the median HbAlc level was within normal ranges for $87 \%$ of patients at 1- and 3-year followup.

The mechanism of T2DM resolution after sleeve gastrectomy is widely discussed. Some believe that hormonal regulation plays a key role $[20,34]$. In this regard, Peterli et al. measured higher GLP-1 levels following ISG [35]. This stimulation may be caused by a progressed gastrointestinal passage after sleeve gastrectomy. Comparison of small bowel transit time from patients after ISG to controls showed a significant reduction in transit time from $298.1 \pm 9.2$ to $199 \pm 65.7$ minutes [36]. Also, an increase on PYY can be seen after ISG with possible amelioration of glucose control $[37,38]$.

Overall, ISG is a restrictive operation. Weight loss is greatest in the first months after operation, so it does not seem surprising to see rapid amelioration of T2DM. In a recent, study performing LSG in nonmorbidly obese T2DM patients resulted in a resolution of T2DM in up to $50 \% 1$ year after operation. The effect is related more to a decrease of insulin resistance because of calorie restriction and weight loss rather than to an increase of insulin secretion [19].

Besides weight loss, the amelioration of T2DM most likely seems to create the condition for resolution of NASH after ISG. Mathurin et al. proposed that remaining insulin resistance is the main risk factor for nonresolution of NASH after obesity surgery [28]. Furthermore, insulin sensitisers such as Pioglitazone and Rosiglitazone can be applied as medical treatment for NASH $[10,11]$. The GLP-1 agonist Exenatide could be administered with success; however, data are scarce $[39,40]$.

Changes in adipokine profiling add to this effect, especially given the fact that only 14 of 87 patients with NASH had HbA1c levels above $6.5 \%$. For adiponectin, it was demonstrated that lower adiponectin levels are associated with increased severity of hepatic inflammation. A repletion of adiponectin in mice resulted in significant improvements in steatosis and inflammation $[41,42]$. For TNF- $\alpha$ elevated plasma concentrations, as can be detected in obese patients, mediated hepatic injury by inhibition of mitochondrial electron transport and release of reactive oxygen species that stimulate lipid peroxidation [43]. Clearly more research is needed to further clarify these correlations.

\section{Conclusion}

ISG conducted in 236 patients resulted in a significant and sustained weight loss comparable to data presented by others. Although a significant difference was seen between NASH patients and patients with normal histology, NASH patients showed a highly significant loss of body weight. For this subgroup, amelioration of HbAlc levels and equally important amelioration of liver histology could be demonstrated. ISG can be successfully performed in NASH patients and, as a fairly easy and safe bariatric intervention to conduct, it can be particularly recommended for this subgroup.

\section{Acknowledgment}

W. K. Karcz and D. Krawczykowski are contributed equally to this work.

\section{References}

[1] R. T. Wang, R. L. Koretz, and H. F. Yee, "Is weight reduction an effective therapy for nonalcoholic fatty liver? A systematic review," American Journal of Medicine, vol. 115, no. 7, pp. 554559, 2003.

[2] G. C. Farrell and C. Z. Larter, "Nonalcoholic fatty liver disease: from steatosis to cirrhosis," Hepatology, vol. 43, no. 2, supplement 1, pp. S99-S112, 2006.

[3] J. Edmison and A. J. McCullough, "Pathogenesis of nonalcoholic steatohepatitis: human data," Clinics in Liver Disease, vol. 11, no. 1, pp. 75-104, 2007.

[4] E. Bugianesi, N. Leone, E. Vanni et al., "Expanding the natural history of nonalcoholic steatohepatitis: from cryptogenic cirrhosis to hepatocellular carcinoma," Gastroenterology, vol. 123, no. 1, pp. 134-140, 2002.

[5] D. M. Torres and S. A. Harrison, "Diagnosis and therapy of nonalcoholic steatohepatitis," Gastroenterology, vol. 134, no. 6, pp. 1682-1698, 2008.

[6] V. Ratziu, F. Charlotte, A. Heurtier et al., "Sampling variability of liver biopsy in nonalcoholic fatty liver disease," Gastroenterology, vol. 128, no. 7, pp. 1898-1906, 2005.

[7] J. R. Lewis and S. R. Mohanty, "Nonalcoholic fatty liver disease: a review and update," Digestive Diseases and Sciences, vol. 55, no. 3, pp. 560-578, 2010. 
[8] S. E. Wozniak, L. L. Gee, M. S. Wachtel, and E. E. Frezza, "Adipose tissue: the new endocrine organ? a review article," Digestive Diseases and Sciences, vol. 54, no. 9, pp. 1847-1856, 2009.

[9] J. P. Després, R. Ross, G. Boka, N. Alméras, and I. Lemieux, "Effect of rimonabant on the high-triglyceride/low-HDLcholesterol dyslipidemia, intraabdominal adiposity, and liver fat the ADAGIO-lipids trial," Arteriosclerosis, Thrombosis, and Vascular Biology, vol. 29, no. 3, pp. 416-423, 2009.

[10] G. P. Aithal, J. A. Thomas, P. V. Kaye et al., "Randomized, placebo-controlled trial of pioglitazone in nondiabetic subjects with nonalcoholic steatohepatitis," Gastroenterology, vol. 135, no. 4, pp. 1176-1184, 2008.

[11] B. A. Neuschwander-Tetri, E. M. Brunt, K. R. Wehmeier, D. Oliver, and B. R. Bacon, "Improved nonalcoholic steatohepatitis after 48 weeks of treatment with the PPAR- $\gamma$ ligand rosiglitazone," Hepatology, vol. 38, no. 4, pp. 1008-1017, 2003.

[12] A. Uygun, A. Kadayifci, A. T. Isik et al., "Metformin in the treatment of patients with non-alcoholic steatohepatitis," Alimentary Pharmacology and Therapeutics, vol. 19, no. 5, pp. 537-544, 2004.

[13] M. Ekstedt, L. E. Franzén, U. L. Mathiesen, M. Holmqvist, G. Bodemar, and S. Kechagias, "Statins in non-alcoholic fatty liver disease and chronically elevated liver enzymes: a histopathological follow-up study," Journal of Hepatology, vol. 47, no. 1, pp. 135-141, 2007.

[14] J. P. Després, A. Golay, and L. Sjöström, "Effects of rimonabant on metabolic risk factors in overweight patients with dyslipidemia," New England Journal of Medicine, vol. 353, no. 20, pp. 2121-2134, 2005.

[15] P. Marceau, S. Biron, R. A. Bourque, M. Potvin, F. S. Hould, and S. Simard, "Biliopancreatic diversion with a new type of gastrectomy," Obesity Surgery, vol. 3, no. 1, pp. 29-35, 1993.

[16] J. P. Regan, W. B. Inabnet, M. Gagner, and A. Pomp, "Early experience with two-stage laparoscopic Roux-en-Y gastric bypass as an alternative in the super-super obese patient," Obesity Surgery, vol. 13, no. 6, pp. 861-864, 2003.

[17] S. M. Han, W. W. Kim, and JI. H. Oh, "Results of laparoscopic sleeve gastrectomy (LSG) at 1 year in morbidly obese Korean patients," Obesity Surgery, vol. 15, no. 10, pp. 1469-1475, 2005.

[18] S. Kuesters, G. Marjanovic, and W. K. Karcz, "Redo operations after bariatric and metabolic surgery," Zentralblatt fur Chirurgie, vol. 134, no. 1, pp. 50-56, 2009.

[19] W. J. Lee, K. H. Ser, K. Chong et al., "Laparoscopic sleeve gastrectomy for diabetes treatment in nonmorbidly obese patients: efficacy and change of insulin secretion," Surgery, vol. 147, no. 5, pp. 664-669, 2010.

[20] J. Vidal, A. Ibarzabal, F. Romero et al., “Type 2 diabetes mellitus and the metabolic syndrome following sleeve gastrectomy in severely obese subjects," Obesity Surgery, vol. 18, no. 9, pp. 1077-1082, 2008.

[21] D. Nocca, D. Krawczykowsky, B. Bomans et al., "A prospective multicenter study of 163 sleeve gastrectomies: results at 1 and 2 years," Obesity Surgery, vol. 18, no. 5, pp. 560-565, 2008.

[22] E. M. Brunt, C. G. Janney, A. M. Di Bisceglie, B. A. Neuschwander-Tetri, and B. R. Bacon, "Nonalcoholic steatohepatitis: a proposal for grading and staging the histological lesions," American Journal of Gastroenterology, vol. 94, no. 9, pp. 2467-2474, 1999.

[23] N. Basso et al., "Laparoscopic sleeve gastrectomy as first stage or definitive intent in 300 consecutive cases," Surgical Endoscopy. In press.
[24] E. Menenakos, K. M. Stamou, K. Albanopoulos, J. Papailiou, D. Theodorou, and E. Leandros, "Laparoscopic sleeve gastrectomy performed with intent to treat morbid obesity: a prospective single-center study of 261 patients with a median follow-up of 1 year," Obesity Surgery, vol. 20, no. 3, pp. 276282, 2010.

[25] R. A. Weiner, S. Weiner, I. Pomhoff, C. Jacobi, W. Makarewicz, and G. Weigand, "Laparoscopic sleeve gastrectomy-influence of sleeve size and resected gastric volume," Obesity Surgery, vol. 17, no. 10, pp. 1297-1305, 2007.

[26] R. Caiazzo, L. Arnalsteen, M. Pigeyre et al., "Long-term metabolic outcome and quality of life after laparoscopic adjustable gastric banding in obese patients with type 2 diabetes mellitus or impaired fasting glucose," British Journal of Surgery, vol. 97, no. 6, pp. 884-891, 2010.

[27] P. R. Schauer, B. Burguera, S. Ikramuddin et al., "Effect of laparoscopic Roux-en Y gastric bypass on type 2 diabetes mellitus," Annals of Surgery, vol. 238, no. 4, pp. 467-485, 2003.

[28] P. Mathurin, F. Gonzalez, O. Kerdraon et al., "The evolution of severe steatosis after bariatric surgery is related to insulin resistance," Gastroenterology, vol. 130, no. 6, pp. 1617-1624, 2006.

[29] R. Singhal, M. Kitchen, S. Bridgwater, and P. Super, "Metabolic outcomes of obese diabetic patients following laparoscopic adjustable gastric banding," Obesity Surgery, vol. 18, no. 11, pp. 1400-1405, 2008.

[30] J. B. Dixon, P. S. Bhathal, and P. E. O'Brien, "Weight loss and non-alcoholic fatty liver disease: falls in gamma-glutamyl transferase concentrations are associated with histologic improvement," Obesity Surgery, vol. 16, no. 10, pp. 1278-1286, 2006.

[31] A. Ulitsky, A. N. Ananthakrishnan, R. Komorowski et al., "A noninvasive clinical scoring model predicts risk of nonalcoholic steatohepatitis in morbidly obese patients," Obesity Surgery, vol. 20, no. 6, pp. 685-691, 2010.

[32] A. Ulitsky, A. N. Ananthakrishnan, R. Komorowski et al., "A noninvasive clinical scoring model predicts risk of nonalcoholic steatohepatitis in morbidly obese patients," Obesity Surgery, vol. 20, no. 6, pp. 685-691, 2010.

[33] N. C. Chavez-Tapia, F. I. Tellez-Avila, T. Barrientos-Gutierrez, N. Mendez-Sanchez, J. Lizardi-Cervera, and M. Uribe, "Bariatric surgery for non-alcoholic steatohepatitis in obese patients," Cochrane Database of Systematic Reviews, no. 1, p. CD007340, 2010.

[34] F. Abbatini, M. Rizzello, G. Casella et al., "Long-term effects of laparoscopic sleeve gastrectomy, gastric bypass, and adjustable gastric banding on type 2 diabetes," Surgical Endoscopy, vol. 24, no. 5, pp. 1005-1010, 2009.

[35] R. Peterli, B. Wölnerhanssen, T. Peters et al., "Improvement in glucose metabolism after bariatric surgery: comparison of laparoscopic roux-en-Y gastric bypass and laparoscopic sleeve gastrectomy: a prospective randomized trial," Annals of Surgery, vol. 250, no. 2, pp. 234-241, 2009.

[36] S. Shah, P. Shah, J. Todkar, M. Gagner, S. Sonar, and S. Solav, "Prospective controlled study of effect of laparoscopic sleeve gastrectomy on small bowel transit time and gastric emptying half-time in morbidly obese patients with type 2 diabetes mellitus," Surgery for Obesity and Related Diseases, vol. 6, no. 2, pp. 152-157, 2010.

[37] S. N. Karamanakos, K. Vagenas, F. Kalfarentzos, and T. K. Alexandrides, "Weight loss, appetite suppression, and changes in fasting and postprandial ghrelin and peptide-YY levels after Roux-en-Y gastric bypass and sleeve gastrectomy: 
a prospective, double blind study," Annals of Surgery, vol. 247, no. 3, pp. 401-407, 2008.

[38] K. Meirelles, T. Ahmed, D. M. Culnan, C. J. Lynch, C. H. Lang, and R. N. Cooney, "Mechanisms of glucose homeostasis after roux-en-y gastric bypass surgery in the obese, insulin-resistant zucker rat," Annals of Surgery, vol. 249, no. 2, pp. 277-285, 2009.

[39] X. Ding, N. K. Saxena, S. Lin, N. Gupta, and F. A. Anania, "Exendin-4, a glucagon-like protein-1 (GLP-1) receptor agonist, reverses hepatic steatosis in ob/ob mice," Hepatology, vol. 43, no. 1, pp. 173-181, 2006.

[40] M. E. Tushuizen, M. C. Bunck, P. J. Pouwels, J. H. T. van Waesberghe, M. Diamant, and R. J. Heine, "Incretin mimetics as a novel therapeutic option for hepatic steatosis," Liver International, vol. 26, no. 8, pp. 1015-1017, 2006.

[41] G. Targher, L. Bertolini, S. Rodella et al., "Associations between plasma adiponectin concentrations and liver histology in patients with nonalcoholic fatty liver disease," Clinical Endocrinology, vol. 64, no. 6, pp. 679-683, 2006.

[42] A. Xu, Y. Wang, H. Keshaw, L. Y. Xu, K. S. L. Lam, and G. J. S. Cooper, "The fat-derived hormone adiponectin alleviates alcoholic and nonalcoholic fatty liver diseases in mice," Journal of Clinical Investigation, vol. 112, no. 1, pp. 91-100, 2003.

[43] D. Pessayre, B. Fromenty, and A. Mansouri, "Mitochondrial injury in steatohepatitis," European Journal of Gastroenterology and Hepatology, vol. 16, no. 11, pp. 1095-1105, 2004. 


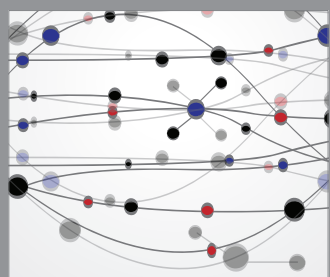

The Scientific World Journal
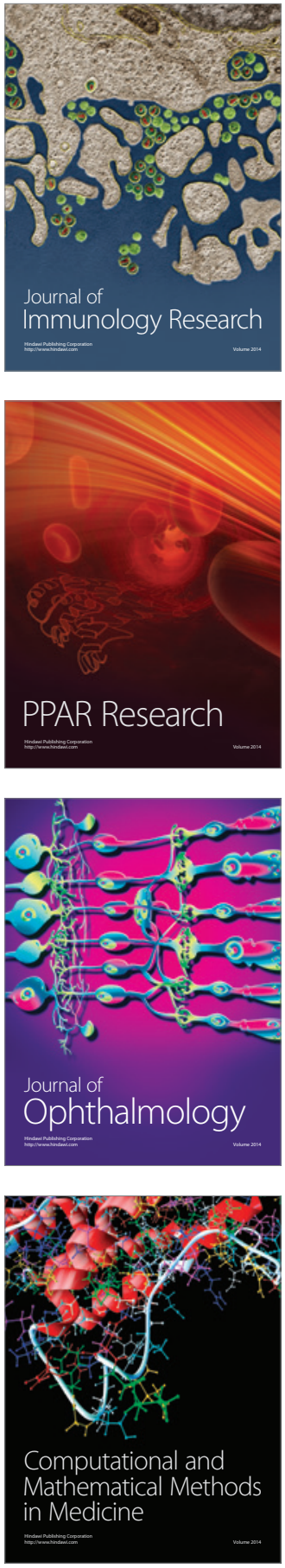

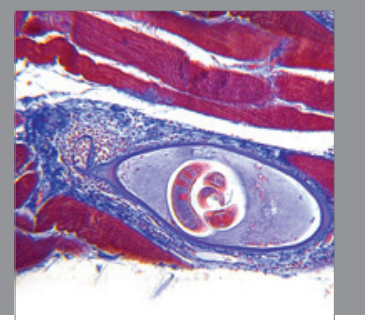

Gastroenterology

Research and Practice
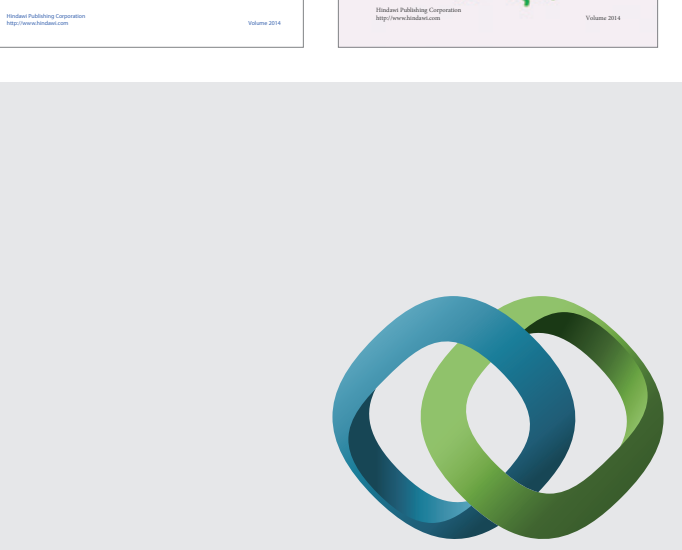

\section{Hindawi}

Submit your manuscripts at

http://www.hindawi.com
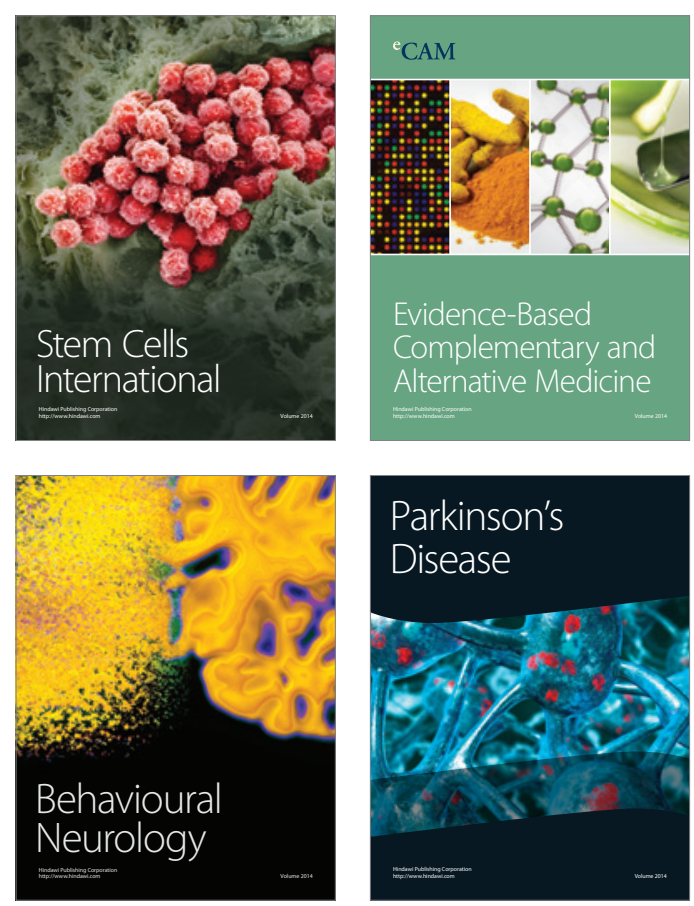

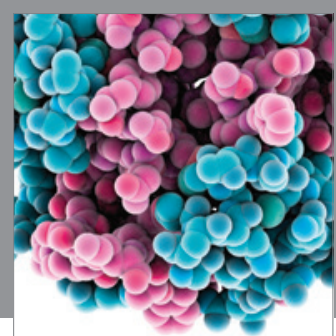

Journal of
Diabetes Research

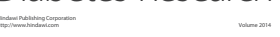

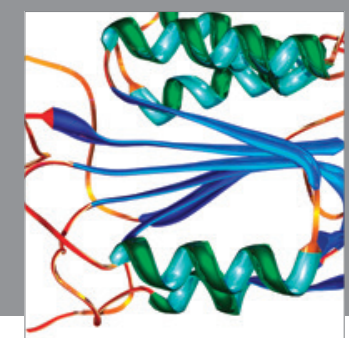

Disease Markers
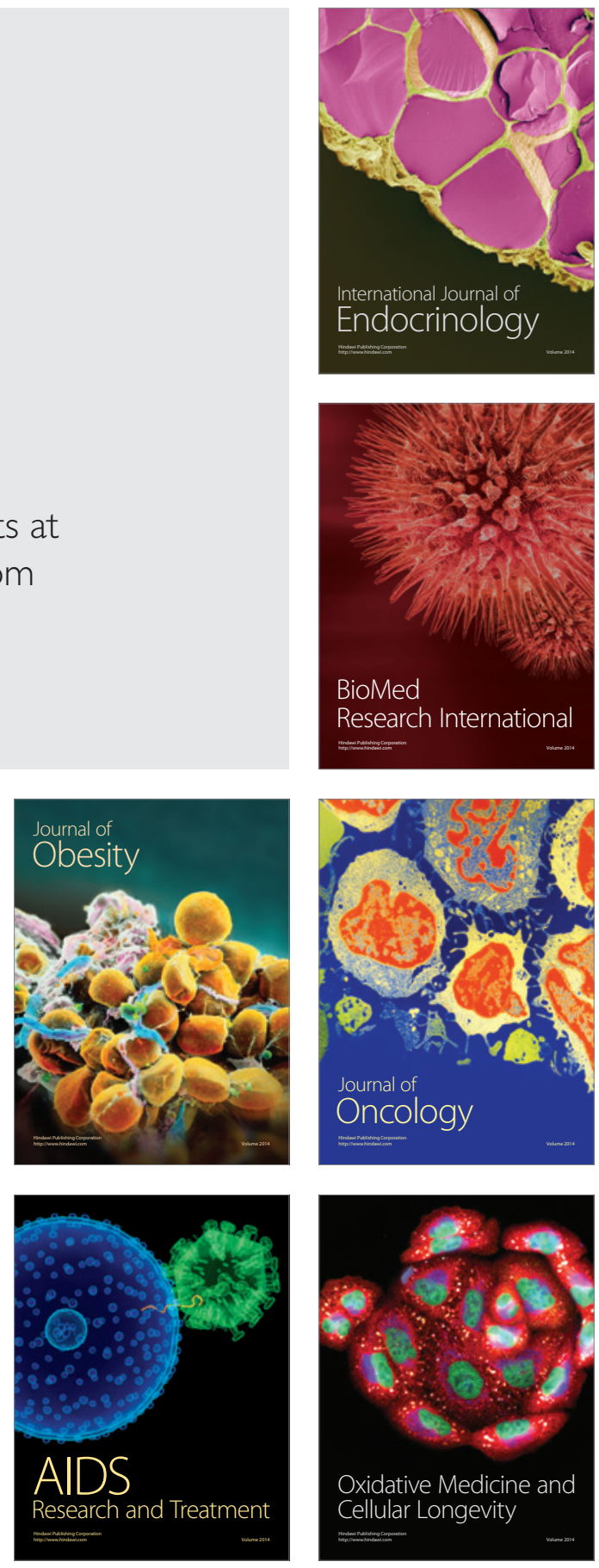